\title{
Mosambik am Scheideweg - die Verfassung von 2004
}

\author{
Von Dietrich Nelle, Bonn
}

\section{Einleitung}

Die besonderen Verbindungen zwischen Mosambik und Deutschland beruhen noch heute auf der Anfangszeit der staatlichen Unabhängigkeit Mosambiks. Bereits im Unabhängigkeitsjahr 1975 nahmen Mosambik und die DDR diplomatische Beziehungen auf; seit 1977 kam es auch zu einer engen wirtschaftlichen Zusammenarbeit, in deren Verlauf über 20.000 Vertragsarbeiter in die DDR zuwanderten. Hieraus ergeben sich vielfältige fortbestehende persönliche Verbindungen. Wirtschaftlich hingegen hat Deutschland bislang kaum an dem bemerkenswerten Stabilisierungsprozess partizipieren können, welchen Mosambik seit 1989 vollzieht. Die Bundesrepublik ist der fünftgrößte Abnehmer von Rohstoffen und landwirtschaftlichen Produkten des Landes, exportiert aber selber kaum dorthin. Im Folgenden werden die mit dem Transformationsprozess des südostafrikanischen Landes verbundenen Verfassungsreformen sowie die diesen zugrundeliegenden gesellschaftlichen und wirtschaftlichen Entwicklungen beleuchtet.

\section{Allgemeine Entwicklungen \\ a) Die erste Phase der Transformation}

Das Land wird seit seiner Unabhängigkeit von der portugiesischen Kolonialherrschaft 1975 durch die 1962 als Freiheitsbewegung gegründeten FRELIMO $^{1}$ regiert. Die nächsten anderthalb Jahrzehnte waren durch ein marxistisch ausgerichtetes Einparteienregime geprägt. Galten die ersten Jahre nach der Unabhängigkeit noch als Phase der Konsolidierung, so zeigten sich spätestens Anfang der 80er Jahre deutliche Anzeichen für eine heraufziehende Krise und einen von beiden Seiten äußerst grausam geführten Bürgerkrieg, welcher nach unabhängigen Schätzungen rund 1 Mio. Menschen das Leben kosten und zahlreiche weitere aus ihrer Heimat vertreiben sollte ${ }^{2}$. Hierbei standen dem Staat und der

1 Frente de Libertação de Moçambique (Befreiungsfront von Mosambik).

2 Bertelsmann Transformation Index 2003, Länderbericht 2003, Gütersloh 2004, 2. 
FRELIMO die 1976 gegründete Rebellenbewegung RENAMO ${ }^{3}$ gegenüber, welche vor allem im Norden und der Landesmitte Unterstützung fand. Zugleich zeigte sich immer mehr das Scheitern des von der Regierung verfolgten zentralwirtschaftlichen Konzepts. Der im sozialistischen Entwicklungsmodell gänzlich vernachlässigte Bereich der Subsistenzlandwirtschaft führte zu weiterer Marginalisierung und wachsender Unzufriedenheit dieses Bevölkerungsteils. Zwangsumsiedlungen und die Zerstörung traditioneller Strukturen schwächten die Unterstützung für die Regierung weiter und schufen gleichzeitig für die RENAMO eine gewisse Legitimationsbasis in der Bevölkerung. Die enge Verflechtung der mosambikanischen Entwicklung mit den Konflikten in Nord-Rhodesien/Zimbabwe und in Südafrika erschwerte die Lösung der Probleme in Mosambik zusätzlich.

\section{b) Die zweite Phase der Transformation}

Eine erste grundlegende Wende trat 1989 mit dem V. Parteikongress der FRELIMO ein. Dort wurde die Trennung von Staat und Partei beschlossen und der Marxismus-Leninismus aus den Parteistatuten gestrichen. Dieser Wandel ermöglichte Friedensverhandlungen, welche 1990 in Rom begannen ${ }^{4}$. Parallel hierzu setzte die FRELIMO-Regierung ihre Reformen fort und entzog so der RENAMO die Rechtfertigung für die Fortsetzung des bewaffneten Kampfes. Ende November 1990 trat eine neue mosambikanische Verfassung in Kraft, welche ohne Beteiligung der RENAMO ausgearbeitet worden war, zugleich aber deren wichtigste Forderungen nach Verankerung von Rechtsstaatlichkeit und Demokratisierung einlöste. Daraufhin kam es im Jahre 1992 in Rom zu einem mit Hilfe der Kirchen vermittelten Friedensschluss. Dieser bildete die Grundlage für die weitere Demokratisierung mit einem Mehrparteiensystem und öffnete den Weg für die wirtschaftliche Entwicklung des Landes. Die RENAMO konstituierte sich als politische Partei und gliederte sich, wenngleich aus einer Position der Schwäche heraus, in den Demokratisierungsprozess ein.

Erste demokratische Wahlen konnten im Jahr 1994 stattfinden. Diese wurden von ausländischen Beobachtern als im wesentlichen frei und ordnungsgemäß charakterisiert. Entscheidend für den Erfolg dieser Wahlen war vor allem die Akzeptanz des Wahlergebnisses durch alle Beteiligten und damit eine weitere Konsolidierung des Friedensprozesses. Sie bestätigten die FRELIMO als Regierungspartei, während die nur wenig schwächere RENAMO auf einen späteren friedlichen Machtwechsel hoffen durfte. 
Die weiterhin bestehenden Schwierigkeiten traten bei den Kommunalwahlen 1998 deutlich zutage. Obgleich der Wahlgang als solcher erneut als frei und ordnungsgemäß charakterisiert werden konnte, führte er aufgrund eines Boykotts der Wahlen durch die RENAMO und einer Wahlbeteiligung von lediglich 14,58\% zu keinem überzeugenden Ergebnis.

In den zweiten Parlamentswahlen 1999 konnte die FRELIMO abermals die Parlamentsund Präsidentschaftswahlen knapp für sich entscheiden ${ }^{5}$. Auch diese Wahlen wurden von unabhängigen Beobachtern als im wesentlichen ordnungsgemäß qualifiziert. Nichtsdestotrotz sah sich die Opposition um ihren Wahlsieg betrogen und weigerte sich - unter Verweis auf Unregelmäßigkeiten bei der Auszählung und Prozessierung der Stimmen - das Wahlergebnis anzuerkennen.

\section{c) Gegenwärtige Lage}

Im Gegensatz zu fast allen anderen Ländern in der Region hat sich in Mosambik ein ZweiParteien-System herausgebildet, in dem sowohl die Regierungspartei FRELIMO als auch die größte Oppositionspartei RENAMO über in etwa gleich große Wählerpotentiale verfügen $^{6}$. Die übrigen Oppositionsparteien spielen nur eine untergeordnete Rolle. Bei den ersten Kommunalwahlen unter Beteiligung der Opposition Ende 2003 verlor die FRELIMO fünf von 33 Städten und musste erstmals beweisen, dass sie bereit ist, Macht abzugeben. Dennoch sichern die Nachwirkungen des einstigen Einheitsstaates und die Beibehaltung eines Präsidialsystems einstweilen die Vorherrschaft der Regierungspartei FRELIMO. Obgleich die begrenzte Parlamentsmehrheit in gewissem Umfang Zugeständnisse an die Opposition erfordert, sind Exekutive, Legislative und Judikative nach wie vor durch die FRELIMO dominiert.

Die dritten freien Parlaments- und Präsidentschaftswahlen fanden am 1./2.12.2004 gleichzeitig mit dem Referendum über die neue Staatsverfassung statt ${ }^{7}$. Nach dem offiziellen Wahlergebnis $^{8}$ konnte FRELIMO die Zahl seiner Parlamentsmandate von 133 auf 160 ausbauen, während RENAMO im gleichen Umfang auf nunmehr 90 Sitze in dem 250köpfigen Gremium fiel. RENAMO behauptete damit trotz dieses Einbruchs eine klare

Mozambique: Election Could be Close-Run Race, VN Integrated Regional Information Networks vom 3.12.2004. 
Sperrminorität gegenüber verfassungsändernden Beschlüssen. Bei der Präsidentschaftswahl verteilten sich die Stimmen ganz ähnlich. Hier siegte der statt des nicht mehr angetretenen bisherigen Präsidenten Chissano der FRELIMO-Kandidat Guebuza mit 63,7 \% der Stimmen gegenüber 31,7 \% für Oppositionsführer Dakhlama. Auf die weiteren Bewerber entfielen jeweils unter $3 \%$. Die Wahlen wurden von internationalen Beobachtern trotz gewisser Mängel wiederum als im wesentlichen frei und ordnungsgemäß bezeichnet ${ }^{9}$. Eine mögliche Erklärung für die offensichtlich auf mangelnde Mobilisierung des eigenen Wählerpotentials zurückzuführenden Verluste der RENAMO könnte in der unterproportionalen Teilhabe ihrer Hochburgen im Norden des Landes an den Früchten des Konsolidierungsprozesses begründet liegen. Auch nach den jüngsten Wahlen weigerte sich die Führung der RENAMO erneut, ihre Niederlage einzugestehen ${ }^{10}$ und provozierte mit diesem Kurs zugleich die Gefahr einer inneren Spaltung der nach wie vor größten Oppositionspartei ${ }^{11}$.

\section{Prozess der Verfassungsgebung}

Mit dem Tag der Unabhängigkeit trat am 24.7.1975 eine erste, von der FRELIMO ausgearbeitete Verfassung in Kraft ${ }^{12}$. Diese schrieb die Rolle der FRELIMO als führende Kraft in Staat und Gesellschaft und eine Personalunion von Partei- und Staatspräsident fest und sanktionierte damit das Einparteiensystem der FRELIMO. Die noch unilateral von der FRELIMO reformierte und Ende November 1990 in Kraft getretene zweite mosambikanische Verfassung gestaltete die bisherige Ordnung bereits grundlegend um. Insbesondere sah sie eine Garantie individueller Grundrechte wie Glaubens-, Meinungs-, und Versammlungsfreiheit, Parteienpluralismus, Unabhängigkeit der Gerichte, freie und geheime Wahlen sowie die Direktwahl des Staatspräsidenten vor.

Im Jahr 1999 erfolgte ein erster Anlauf für eine grundlegende Reform dieser Verfassung durch die beiden ehemaligen Konfliktparteien gemeinsam, welche bei diesem Vorhaben aufeinander angewiesen waren, da sie nur zusammen die erforderlichen parlamentarischen Mehrheiten erreichen konnten ${ }^{13}$. Zunächst in der Diskussion befindliche weitreichende Reformen des politischen Systems erwiesen sich so als undurchführbar, und die Reform musste sich auf zwischen beiden Seiten konsensfähige Fragen beschränken. Im Parlament

UN-Integrated Regional Information Network v 21.12.2004. 
ermöglichte dies die einstimmige Verabschiedung ${ }^{14}$. Nach einem erwartungsgemäß positiven Referendum im Dezember 2004 sind die Änderungen am 17.12.2004 in Kraft getreten.

\section{Struktur der neuen Verfassung}

Trotz der sich aus dem Verfassungsprozess ergebenden Einschränkungen ist der Umfang der Verfassung im Vergleich zu ihren Vorgängerinnen erheblich gewachsen und umfasst nunmehr 306 statt bisher 217 Artikel in der Verfassung von 1990 bzw nur 71 Artikeln der Verfassung von 1974. Nachdem die Verfassung von 1974 über weite Strecken einen mehr programmatischen als rechtlich-regelnden Charakter trug, brachte die Verfassung von 1990 in vielen Fragen erstmals konkrete Regelungen. Die erneute Steigerung des Umfangs in der Verfassung von 2004 resultiert vor allem aus einem größeren Detailgrad der einzelnen Regelungen; erweitert wurden insbesondere die Abschnitte über Grundrechte (Art 35 ff), über die wirtschaftliche und soziale Grundordnung (Art 96 ff) sowie über das Parlament (Art 168 ff). Neu eingefügt wurden lediglich die Abschnitte über die öffentliche Verwaltung (Art 249 ff.), das Polizeiwesen (Art 254 ff), den Ombudsman (Art 256 ff) sowie den Notstand (Art 282 ff). Die größere Bedeutung des Abschnittes über Grundrechte wird zudem dadurch unterstrichen, dass er in der Textreihenfolge weiter nach vorne rückt.

\section{Freiheitsrechte, Justizgewährleistung, politische Teilhabe}

Trotz des umfangreichen Grundrechtsschutzes durch die Verfassung von 1990 wurden bürgerliche Freiheitsrechte insbesondere durch Gesetzesmissachtungen staatlicher Sicherheitsorgane auch weiterhin eingeschränkt ${ }^{15}$. Besonders gravierende Fälle waren die gewaltsame Auflösung von Demonstrationen der Opposition und der Erstickungstod von 119 Häftlingen im Gefängnis von Montepuez im November 2000. Nach Ansicht unabhängiger Beobachter spielten dabei allerdings weniger politische Beweggründe eine Rolle als mangelnde Kenntnis der Menschenrechte bei der Polizei und Korruption ${ }^{16}$.

Vor dem Hintergrund dieser internen Problematik, aber auch im Hinblick auf die ausländische Öffentlichkeit bildet die Ausweitung der Ausführungen zum Grundrechtsschutz (Art 35 ff.) einen der Schwerpunkte der Novelle. Das Verbot von Todesstrafe, Folter sowie

Constitutional Amendments Finally Passed, Agencia de Informação de Moçambique vom 16.11.2004.

15

Crause/Huber, Menschenrechte in Mosambik, MenschenRechtsMagazin 3/1998.

16

Bertelsmann Transformation Index 2003, Länderbericht 2003, Gütersloh 2004, 9; Renamo Deputy

Detained, Agencia de Informação de Moçambique vom 2.12.2004. 
grausamer und unmenschlicher Behandlung wird fortgeschrieben (Art 40). Hinzu tritt neu das Verbot auch der lebenslänglichen Freiheitsstrafe (Art 61 (1). Die Grundsätze der Öffentlichkeit von Gerichtsverfahren (Art 65 (2)), der Unschuldsvermutung (Art 59 (2)) sowie des Verbots der Doppelverfolgung (Art 59 (3)) erhalten ebenso Verfassungsrang wie das Verwertungsverbot für durch Folter, Zwang oder unerlaubtes Abhören erlangte Beweismittel (Art 65 (3)), der Anspruch auf den gesetzlichen Richter (Art 65 (4)), das Recht auf freie Wahl des Verteidigers (Art 62 (2)) sowie der Schutz des Anwaltsgeheimnisses (Art 63). Neu ist ferner ein Datenschutzgrundrecht, welches die Erfassung von Bürgern im Hinblick auf deren politische und religiöse Überzeugungen oder ihre Partei- und Gewerkschaftszugehörigkeit verbietet (Art 70 (1)). Die Bürger haben jetzt ein verfassungsmäßiges Recht auf Einsichtnahme in sie betreffende Verwaltungsakten sowie auf Berichtigung etwaiger Fehler (Art 70 (4)).

Die Grundrechte Demonstrations- (Art 51) und Vereinigungsfreiheit (Art 52), freie Gründung politischer Parteien (Art 53) und Freizügigkeit (Art 55) wurden aus der bisherigen Verfassung übernommen. Die Religionsfreiheit wurde durch ein Recht auf Kriegsdienstverweigerung aus Gewissensgründen ergänzt (Art 54 (4)). Kaum praktikabel dürfte dagegen die neu eingeführte Zivildienstpflicht für alle diejenigen, welche nicht in der Armee dienen Art 267 (3)) $\operatorname{sein}^{17}$.

Die Einschränkung von Grundrechten durch Ausrufung des Notstandes wurde erschwert (Art 282 ff.).

\section{Pressefreiheit}

Die Pressefreiheit wird bereits durch die frühere Verfassung garantiert (Art 74). Die Medien machen einen lebhaften Gebrauch von ihr; allerdings stehen die elektronischen Medien auch weiterhin unter starkem staatlichen Einfluss. Dennoch ist dank des Erscheinens von über 30 Zeitungen eine Meinungsvielfalt zumindest in der Hauptstadt Maputo weitgehend gewährleistet ${ }^{18}$. Schwieriger ist die Zugänglichkeit von Informationen auf dem Lande. Die regierungsnahe Zeitung Noticias ist eines der wenigen in allen Provinzen vertriebenen Periodica. Unabhängige Informationsvermittlung leisten vor allem per Fax vertriebene Blätter und die Wochenzeitungen Savana und Demos. Der staatliche Rundfunksender Radio Moçambique, der als einziges Medium landesweit auch in lokalen Spra- 
chen sendet, steht im Ruf einer qualitativ hochwertigen und einigermaßen unabhängigen Berichterstattung.

Durch die neue Verfassung wird der Grundrechtsschutz für die Meinungs- und Pressefreiheit ausgeweitet. Dies geschieht insbesondere dadurch, dass Teile des bisherigen Pressegesetzes Verfassungsrang erhalten (Art 48 ff.). Die Verfassung garantiert nun Wiedergabe und Wettstreit unterschiedlicher politischer Richtungen in den staatlichen Medien. Außerdem werden die Journalisten dem Einfluss von Regierung, Verwaltung und anderen politischen Kräften entzogen. Die missbrauchsanfällige Klausel der bisherigen Verfassung, dass die Berichterstattung die Aufträge der Außenpolitik und der nationalen Verteidigung zu achten hatte (Art 74 (4) aF), wurde gestrichen.

\section{Wirtschaftliche, soziale und kulturelle Grundrechte}

Aus der bisherigen Verfassung übernommen wurde der Schutz von Eigentum (Art 82), Erbrecht (Art 83), Arbeit (Art 84), guten Arbeitsbedingungen (Art 85), gewerkschaftlicher Betätigung (Art 86), Streik (bei ausdrücklichem Verbot der Aussperrung, Art 87)), Bildung (Art 88), geistiger Schöpfung (Art 94), Gesundheit (Art 89), Sport (Art 93), Umwelt (Art 89) sowie von behinderten und alten Menschen (Art 95).

Die Erweiterungen des Grundrechtsschutzes in diesem Bereich dienten nicht der Ausdehnung individueller Freiheiten, sondern ausschließlich der Verstärkung sozialer Teilhabe. So wurde ein Anspruch aller Bürger auf würdige Unterkunft geschaffen, für welche der Staat entsprechend der nationalen Wirtschaftsentwicklung die Voraussetzungen zu schaffen hat. Neu eingefügt wurde auch ein Verbraucherschutzgrundrecht (Art 92). Danach haben Verbraucher Anspruch auf Waren und Dienstleistungen von guter Qualität, auf zutreffende Information sowie auf Schutz ihrer Gesundheit und Sicherheit. Neu ist des Weiteren, dass versteckte, indirekte und täuschende Werbung durch die Verfassung selber verboten wird. Verbraucherschutzvereinigungen haben in solchen Fällen die Befugnis, zum Schutz ihrer Mitglieder Klage zu erheben. Eine bedenklich weit gefasste Popular- und Verbandsklage wird zudem Einzelpersonen und Vereinigungen zugebilligt, um insbesondere Schadenersatzansprüche durchzusetzen sowie Schädigungen der öffentlichen Gesundheit, öffentlichen Vermögens oder öffentlichen Kulturgutes abzuwehren (Art 81). Welche Rückwirkungen sich hieraus auf das Wirtschaftsgeschehen ergeben werden, bleibt einstweilen abzuwarten.

Neu hinzugekommen ist ferner ein Kinderschutzrecht (Art 47). Danach haben Kinder Anspruch auf Schutz und Sorge sowie in den sie betreffenden Angelegenheiten auf Gehör entsprechend ihrem Alter und ihrer Reife. Alle Handlungen öffentlicher oder privater Ein- 
richtungen, soweit sie Kinder betreffen, haben sich in erster Linie am höheren Kindesinteresse auszurichten.

\section{Staat und Religion}

Vor Erlangung der Unabhängigkeit spielte die katholische Kirche eine zentrale Rolle im Lande. Mit der Unabhängigkeit transformierte sich Mosambik von einem Konfessionsstaat, in dem die Katholische Kirche eine bevorzugte Position einnahm, in einen laizistischen Staat. Die Kirchen blieben jedoch weiterhin einflussreich, obwohl nur 30-40 \% der Bevölkerung Christen sind. Rund die Hälfte der Bevölkerung neigt Naturreligionen zu, die verbleibenden 10-20 \% bekennen sich zum Islam ${ }^{19}$. Insbesondere die westlich geprägten und im Conselho de Cristão de Moçambique (CCM) zusammengeschlossenen protestantischen Kirchen sowie die Katholische Kirche spielten eine wichtige Rolle in der Initiierung des Friedensprozesses.

Auch die neue Verfassung betont die laizistische Ausrichtung des Staates (Art 9 (1)). Gleichzeitig wird jedoch in der Neuregelung der bisherige antiklerikale Unterton vermieden und die Tragweite der Bestimmung dahingehend klar gestellt, dass damit die Trennung von Staat und Kirche gemeint sei (Art 9 (2)). Zugleich wird jetzt auch die Freiheit der Gründung und Betätigung von Religionsgemeinschaften betont (Art 9 (3)).

\section{Staatsangehörigkeit}

Eine weitere wichtige Neuerung betrifft das Recht der Staatsangehörigkeit (Art 23 ff) ${ }^{20}$. Neben der Gleichstellung ausländischer Ehepartner und der Einschränkung der bislang rechtstaatlich bedenklich weiten Gründe für deren unfreiwilligen Verlust ist die bedeutsamste Neuerung die praktisch unbegrenzte Zulassung der doppelten Staatsangehörigkeit. Allerdings wird diese so ausgestaltet, dass sie nur zugunsten ausgewanderter Bürger wirkt, welche so weiter an ihr Heimatland gebunden bleiben, während im Inland ausschließlich die mosambikanische Staatsangehörigkeit Bedeutung haben soll. Die Hürden für eine Einbürgerung bleiben hoch; obwohl mit der Zugrundelegung des ius soli als Grundsatz für den originären Erwerb der Staatsangehörigkeit ein im Grundsatz einwanderungsfreundli-

19 Proceminho, República de Moçambique - Ficha de Mercado, Maputo 2002.

20

Constitution: Dual Nationality, Agencia de Informação de Moçambique vom 23.9.2004; vgl Gesetz v 22.5.1972 über die mosambikanische Staatsangehörigkeit; Zitscher, Mosambik: Staatsangehörigkeitsrecht, StAZ 1994, S. 158 ff. 
ches Recht geschaffen wurde. Es wird ein Mindestaufenthalt von zehn Jahren verlangt, welcher sich bei besonderen Personengruppen auf fünf Jahre reduziert.

\section{Staatsorganisation}

a) Allgemeines

Auch nach der Kompetenzverteilung der neuen Verfassung bleibt Mosambik eine zentralistisch strukturierte Präsidialdemokratie. Vorgesehen war insbesondere, die Kompetenzen des Staatspräsidenten drastisch zu beschneiden und einen parlamentarisch verantwortlichen Ministerpräsidenten einzuführen. Bevor diese Änderungen verabschiedet werden konnten, distanzierte sich allerdings die Oppositionspartei RENAMO, deren Stimmen für eine verfassungsändernde Zweidrittel-Mehrheit benötigt wurden, von diesen Plänen. Sie hoffte vielmehr, über einen nach den nächsten Wahlen ggf. von ihr selber gestellten Präsidenten das bislang von der früheren Einheitspartei bestimmte Staatswesen wirksamer umgestalten zu können. Dennoch zeigt sich eine gewisse Auflockerung des Grundsatzes der Gewaltenteilung (Art 134) sowie in vorsichtigen Änderungen in der Stellung der einzelnen Organe.

\section{b) Präsident}

An der Spitze der Exekutive steht der weiterhin in direkter Wahl zu bestimmende Staatspräsident (Art 147). Auch nach der neuen Verfassung fungiert er gleichzeitig als Staatsoberhaupt, Regierungschef und Oberkommandierender der Streitkräfte (Art 146, 266). Er kann nach seinem Gutdünken Minister, Staatssekretäre und Provinzgouverneure einsetzen (Art. 160). Außerdem ernennt er u.a. das Oberkommando von Militär und Polizei, die obersten Richter, den Generalstaatsanwalt, den Leiter der Staatsbank sowie die Rektoren der Hochschulen (Art 160, 161, 226, 239). Seine Stellung wird lediglich insofern eingeschränkt, als ein Amtsenthebungsverfahren neu geregelt wird. Er kann künftig mit zwei Dritteln der Stimmen des Parlaments wegen in Ausübung seines Amtes begangener Straftaten angeklagt werden. In diesem Fall findet ein Verfahren vor dem Obersten Gerichtshof statt, welches im Falle einer Verurteilung zur Amtsenthebung sowie zum dauerhaften Verlust des passiven Wahlrechts führt (Art 153).

Neu ist die Einrichtung eines Staatsrats (Art $164 \mathrm{ff}$ ). Dieses Gremium besteht aus dem Ministerpräsidenten, dem Parlamentspräsidenten, dem Ombudsman, ehemaligen Staatspräsidenten und Parlamentspräsidenten, dem mit der zweithöchsten Stimmenzahl unterlegenen Kandidaten der letzten Präsidentenwahl und elf verdienten Persönlichkeiten, von welchen vier der Präsident und sieben das Parlament ernennen. Es berät den Präsidenten und ist vor jeder Kriegserklärung, Verhängung eines Belagerungs- oder Ausnahmezustandes und der Ausschreibung von Neuwahlen anzuhören. Jedoch besitzt es in keiner dieser Fragen ein 
Vetorecht; letztlich wird vor allem ein Anhörungsrecht der Führung der jeweils größten Oppositionspartei in grundlegenden Fragen gesichert.

\section{c) Regierung}

Der Ministerpräsident wird ebenfalls vom Staatspräsidenten ernannt (Art 160); ihm kommt, wie in Präsidialsystemen üblich, eher eine Koordinierungsfunktion zu. Den Vorsitz im Kabinett führt nicht er, sondern der Staatspräsident, welcher auch die übrigen Regierungsmitglieder bestimmt. Eine echte parlamentarische Verantwortung der Regierung ist nicht gegeben. Lehnt das Parlament das Regierungsprogramm mehrheitlich ab, kann auch nach der neuen Verfassung der Präsident das Parlament auflösen und Neuwahlen ausschreiben (Art 188). Das Regierungsamt ist inkompatibel mit einem Parlamentsmandat sowie mit dem aktiven Dienst in Militär, Diplomatie und Justiz oder der Leitung einer Lokalverwaltung (Art $172(1))$.

\section{d) Parlament}

Seit den ersten freien Wahlen vom Oktober 1994 liegt die gesetzgebende Gewalt beim Parlament $^{21}$. Die Bipolarität der früheren Bürgerkriegsparteien setzt sich heute im Parlament fort. Mehrheits- und Regierungspartei ist die frühere Einheitspartei FRELIMO, die Opposition besteht hauptsächlich aus der aus der gleichnamigen Rebellenbewegung hervorgegangenen RENAMO-Partei. Das Parlament tritt zweimal im Jahr zu ordentlichen und bei Bedarf zu außerordentlichen Sitzungsperioden zusammen.

Das Parlament ist monokameral. Es besteht aus 250 Abgeordneten (Art 170). Der Mandatsverlust bleibt nicht mehr der einfachgesetzlichen Regelung überlassen, sondern wird durch die neue Verfassung unmittelbar geregelt. Außer dem freiwilligen Verzicht zählen zu den Verlustgründen die Verurteilung zu mehr als zwei Jahren Haft wegen einer vorsätzlichen Straftat, der Parteiwechsel sowie das hartnäckige Fehlen bei Parlamentssitzungen (Art 178). Durch das Verbot des Parteiwechsels wird vor allem die dominierende Rolle der beiden Fraktionen vor der Versuchung geschützt, dass deren Angehörige kleinere, konkurrierende Fraktionsgemeinschaften mit Anspruch auf eigene staatliche Unterstützung bilden.

Entsprechend dem französischen Modell ist das Parlament weiter nur in bestimmten Materien gesetzgeberisch tätig; die Rechtsetzung im übrigen wird durch die Regierung wahrgenommen. Durch die neue Verfassung wurde zugleich aber auch die Rolle des Parlaments in diesem Bereich deutlich gestärkt. Im Vergleich zur bisherigen Verfassung wird die Zahl der ausdrücklich dem Parlament vorbehaltenen Kompetenzen von sieben auf 22 gesteigert (Art 
179 (2)). In den übrigen Bereichen ist für die Rechtsetzung der Regierung durch Dekret künftig ein Ermächtigungsgesetz erforderlich (Art 179 (3)). Dieses muss nach Inhalt, Zweck, Umfang und Dauer begrenzt sein (Art 180 (1)). Eine solche Ermächtigung kann nur einmalig genutzt werden, und sie verfällt mit dem Ende der Legislaturperiode (Art 180 (2)). Wenn $15 \%$ der Abgeordneten dies verlangen, bedarf ein solches Dekret der parlamentarischen Bestätigung (Art 181 (1)). Das Parlament erhält außerdem ein Rückholrecht, so dass es die Geltung eines solchen Dekretes auch aufheben oder suspendieren kann (Art 181 (2)).

Eine Stärkung der Stellung des Parlaments bedeutet auch die ausdrückliche Regelung der Durchführung von Volksabstimmungen (Art 136). Zur Abstimmung gestellt werden können alle Fragen von nationalem Interesse. Voraussetzung ist aber, dass das Parlament dies mit der absoluten Mehrheit seiner Mitglieder beantragt und der Staatspräsident zustimmt. Die Rechtmäßigkeit ist vor Abhaltung des Referendums durch den Verfassungsrat zu überprüfen (Art 244 (1)). Eine weitere Stärkung der Parlamentstätigkeit insbesondere der Oppositionsabgeordneten bedeutet auch die neu aufgenommene Regelung der Tätigkeit der Parlamentsfraktionen (Art 196 ff). Diesen werden gewisse Minderheitenrechte sowie Fraktionsräume und -personal zugestanden.

\section{e) Parteien}

Nach der Veränderung der politischen Rahmenbedingungen im gesamten südafrikanischen Raum und der grundlegenden Transformation in Mosambik haben die ehemaligen politischen Etiketten der beiden großen, aus den Bürgerkriegsfraktionen hervorgegangenen Parteien stark an Bedeutung verloren. Ein wichtiger Unterschied besteht aber in der nach wie vor regional (und damit auch ethnisch) äußerst unterschiedlichen Verankerung beider Seiten. Obwohl inzwischen eine Vielzahl von Wahlen erfolgreich durchgeführt werden konnte, bleibt so der Wettstreit der Parteien um politische Ideen und Konzepte nach wie vor weitgehend Theorie.

Vor diesem Hintergrund haben die einschlägigen Verfassungsbestimmungen teilweise mehr programmatischen als beschreibenden Charakter. Wie bereits in der früheren Verfassung werden die Mitwirkung der Parteien an der politischen Willensbildung und der politische Pluralismus festgeschrieben (Art 74 (1)). Parteien müssen intern demokratisch organisiert (Art 74 (2)) sowie landesweit tätig sein und zur patriotischen Gesinnung der Bürger und zum Zusammenhalt der Nation beitragen (Art 75). Die Verwendung religiöser Bezeichnungen oder Symbole ist ausgeschlossen (Art 76). Der Gebrauch von Gewalt als Mittel zur Änderung der politischen oder sozialen Ordnung darf nicht gefördert werden (Art 77). 


\section{f) Ombudsman}

Mit der neuen Verfassung soll nun auch eine institutionelle Vorkehrung zur Eindämmung von Grundrechtsverletzungen und zur Bekämpfung von staatlicher Misswirtschaft, Korruption und Vetternwirtschaft getroffen werden. Das neu geschaffene Amt eines Ombudsmans soll es den Bürgern ermöglichen, wirksamer als bisher gegen solche Missbräuche vorgehen zu können. Bei dem Ombudsman hat es sich um eine unabhängige und unparteiische Persönlichkeit zu handeln (Art 258 (1)). Sie ist vom Parlament mit einer 2/3-Mehrheit zu wählen (Art 257), was bei der gegenwärtigen Stimmenverteilung einen Konsens zwischen beiden politischen Lagern voraussetzt. Der Ombudsman kann falsche Entscheidungen nicht selber abändern. Er hat aber das Recht, eigenständige Untersuchungen durchzuführen und dem Parlament, der Staatsanwaltschaft sowie der zentralstaatlichen und lokalen Verwaltung zu berichten und Vorschläge zu unterbreiten (Art 259); die öffentliche Verwaltung hat mit ihm zusammen zu arbeiten und ihn bei der Durchführung seiner Aufgaben zu unterstützen (Art 260). Außerdem gibt er einmal im Jahr einen Tätigkeitsbericht vor dem Parlament ab (Art 258 (2)).

\section{1. Öffentlicher Dienst}

Nach der Unabhängigkeit wurden die Strukturen des öffentlichen Dienstes nicht grundlegend verändert, sondern nur modifiziert und personell neu besetzt. Ineffizienz und Lethargie portugiesischer Kolonialverwaltungsstrukturen verschärften sich durch Einführung der kommunistischen Prinzipien des "demokratischen Zentralismus" und der doppelten Unterordnung der Verwaltungseinheiten unter Staat und Partei. Insbesondere die Distriktverwaltungen und Lokalbehörden stellen - bedingt durch mangelnde personelle und finanzielle Ressourcen - eher Symbole des Staates denn echte Leistungserbringer für die Bevölkerung dar. Dort wo die staatliche Verwaltung abwesend ist, werden deren Aufgaben zunehmend wieder von traditionellen Führern wahrgenommen, häufig im Kontakt untereinander und mit der Lokalverwaltung. Besonders im Einflussgebiet der RENAMO wurde diese Entwicklung staatlicherseits auch aktiv gefördert.

Auch nach der Transformation pflanzten sich der Mangel an ausgebildetem Personal und finanziellen Ressourcen fort ${ }^{22}$. Nepotismus und Korruption sind feste Bestandteile im institutionellen Gefüge. Mit dem 2002 verabschiedeten Anti-Korruptionsgesetz erfolgte zwar nach langem parlamentarischen Ringen ein sichtbarer Schritt, doch gilt das Gesetz als lückenhaft, und es bestehen Zweifel an der Fähigkeit zur Umsetzung und vor allem am

22

International Monetary Fund, Republic of Mozambique: Poverty Reduction Strategy Paper Progress Report, IMF Country Report 04/135, Washington 2004, 100 ff. 
politischen Willen, auch gegen die herrschende politische Elite vorzugehen. Diese Einstellungen teilen breite Schichten der Bevölkerung. Wie Umfragen zeigen, steht für 58,8 \% der Befragten fest, dass Regierung und Justiz korrupt $\operatorname{sind}^{23} .70,2 \%$ sehen die Polizei als korruptes Staatsorgan.

Die neue Verfassung normiert Grundsätze guter Verwaltungsführung und des Zugangs zur Verwaltungslaufbahn (Art 249 ff.). Auf die Einrichtung eines öffentlichen Verwaltungsrates zur transparenteren Besetzung öffentlicher Ämter wird allerdings verzichtet.

\section{Lokale Verwaltungen}

Mosambik strebt eine Auflockerung der bislang ausgeprägten Zentralisierung an ${ }^{24}$. Im Juni 1998 wurden erstmals in den 33 größeren Orten bzw. Wahlbezirken Kommunalwahlen durchgeführt. Die dabei ermittelten Versammlungen konnten die Spitzen der jeweiligen Lokalverwaltungen bestimmen, auch in der Hauptstadt Maputo. Die Gouverneure, die in den 10 Provinzen (Maputo, Gaza, Inhambane, Sofala, Manica, Tete, Zambezia, Nampula, Niassa, Cabo Delgado) und der Hauptstadt Maputo-Stadt die Zentralgewalt repräsentieren, werden jedoch vom Staatspräsidenten ernannt.

Neu in der Verfassung von 2004 ist, dass gemäß einer Forderung der Opposition künftig auch auf Ebene der Provinzen Parlamente arbeiten sollen, welche im Verhältnis zur jeweiligen Provinzregierung allerdings über ähnlich schwache Kompetenzen verfügen wie das Zentralparlament im Verhältnis zur Zentralregierung (Art 275). Einzelheiten sind durch Gesetz zu regeln. Die ersten Wahlen zu den Provinzversammlungen sind für 2008 geplant $^{25}$.

\section{Traditionelle Autoritäten}

Neben der politischen und administrativen Führung sind auch über die Zeiten der Kolonialherrschaft und der Umgestaltung in einen marxistischen Zentralstaat ${ }^{26}$ traditionelle

Dhlakama Threatens to Take Power, Agencia de Informação de Moçambique vom 29.11.2004; Fandrych, Kommunalreform und Lokalpolitik in Mosambik, Hamburg 2001. 16.11.2004.

26 Art 4 der Verfassung erklärte die Bekämpfung traditioneller Strukturen und Rechtsüberzeugungen sogar zum Verfassungsauftrag. 
Autoritäten lebendig geblieben ${ }^{27}$. Dies gilt sowohl für die Leitung des afrikanischen Familienverbandes durch ein gemeinsames Oberhaupt als auch für die Vermittlung und Streitschlichtung durch lokale Respektspersonen ${ }^{28}$. Angesichts der institutionellen Schwäche des Staates wurden - wie bereits erwähnt - jüngst auch Verwaltungsaufgaben wieder verstärkt von solchen Personen wahrgenommen ${ }^{29}$. Die neue Verfassung erkennt diese Realität ausdrücklich an (Art 118 (1)). Die diesem Prozess freundlich gegenüberstehende RENAMO konnte damit eines ihrer politischen Anliegen realisieren. Politische oder justizielle Sonderrechte für traditionelle Führer leitet die Verfassung hieraus anders als in vielen anderen afrikanischen Ländern allerdings nicht ab. Vielmehr bleibt ihre Rolle auf eine gesetzlich näher $\mathrm{zu}$ beschreibende wirtschaftliche, soziale und kulturelle Mitwirkung beschränkt (Art 118 (2)). Eine mögliche Konkretisierung dieses Handlungsfeldes könnte aus der expliziten Sanktionierung des internen Rechtspluralismus folgen, wonach der Staat die verschiedenen in der mosambikanischen Gesellschaft vorhandenen Normensätze und Mechanismen der Streitschlichtung anzuerkennen hat, soweit sie nicht den Werten und Grundsätzen der Verfassung widersprechen (Art 4). Praktisch besonders bedeutsam sind hier die fortbestehenden traditionellen Bodenrechte (Art 111) ${ }^{30}$. Hinzu kommt, dass auch die Anerkennung und Registrierung der traditionellen und religiösen Eheschließung gesetzlich zu regeln sind (Art $119(4))^{31}$.

\section{Außen- und Sicherheitspolitik}

In der Kooperation mit bilateralen und internationalen Gebern gilt Mosambik als Musterschüler, der zum einen die zur makroökonomischen Zielerreichung auferlegten Konditionen unverzüglich umsetzt und zum anderen durch geschickte diplomatische Darstellungskunst ein Netzwerk an Geberorganisationen mobilisiert und deren Unterstützung auch über längere Zeiträume hinweg aufrechterhält ${ }^{32}$. Selbst die durch den Prozess gegen die Mörder

Bonate, Women's Land Rights in Mozambique, in: An-Na'im/Chandler, Women and Land in Africa, Atlanta 2002, Ziff. III.

Bonate, Women's Land Rights in Mozambique, in: An-Na'im/Chandler, Women and Land in Africa, Atlanta 2002, Ziff. VI.

29

Bonate, Women's Land Rights in Mozambique, in: An-Na'im/Chandler, Women and Land in Africa, Atlanta 2002, Ziff. VI.

Einfachgesetzlich vgl. Gesetz Nr. 19/97 über das Bodenrecht.

31

Damit vollzieht die Verfassung eine bereits durch das neue Familiengesetzbuch (Gesetz Nr. 153/2003 vom 12.12.2003) vollzogenen Änderungen nach; bislang lebte die übergroße Mehrzahl in einer rechtlich nicht anerkannten nichtehelichen Lebensgemeinschaft, vgl. Arnaldo, Ethnicity and Marriage Patterns in Mozambique, African Population Studies 19 (2004), 4 ff.

Bertelsmann Transformation Index 2003, Länderbericht 2003, Gütersloh 2004, 21; Fandrych, Konfliktmanagement und -regelung der Vereinten Nationen in Mosambik, Hamburg 1988. 
des Journalisten Cardoso und diverse Bankenskandale entstandenen Flecken auf Präsident Chissanos weißer Weste trübten nur bedingt seinen internationalen Ruf.

Für die Außenpolitik ist entsprechend dem französischen Modell im wesentlichen der Staatspräsident zuständig, welcher die Leitlinien der Außenpolitik festlegt, internationale Verträge schließt, mosambikanische Diplomaten benennt und entlässt sowie ausländische Diplomaten akkreditiert (Art 162). Die mosambikanische Verfassung enthält darüber hinaus traditionell detaillierte Aussagen zur materiellen Außen- und Sicherheitspolitik des Landes. Als Grundprinzipien werden die wechselseitige Achtung der Souveränität, der territorialen Integrität, Gleichheit, Nichteinmischung in fremde innere Angelegenheiten und der Wechselseitigkeit der Vorteile normiert (Art 17). Aus der Gründungszeit des Landes erklärt sich die Betonung der Solidarität mit anderen afrikanischen Ländern, welche nach Einheit, Freiheit und wirtschaftlichen wie sozialem Fortschritt streben, der besonderen Beziehung zu Staaten, welche Unabhängigkeit und Kontrolle ihrer natürlichen Ressourcen verfolgen sowie des Kampfes für eine gerechte Weltwirtschaftsordnung (Art 19). Die Verpflichtung des Landes auf eine Friedenspolitik wird auf eine generelle Abrüstungspolitik sowie die Umwandlung des Indischen Ozeans in eine entmilitarisierte Zone ausgedehnt (Art 22). Mit dem Ende des Kalten Kriegs wird das Prinzip der Blockfreiheit aufgegeben. Die verfassungsrechtliche Gewährleistung des Asylrechts wird um ein Flüchtlingsstatut ergänzt (Art 20).

Außerdem werden deutlich detailliertere Regelungen zu den Sicherheitsorganen als in der bisherigen Verfassung getroffen. Das Militär wird besonders auf die Verfassung und den Verzicht auf parteipolitische oder andere den nationalen Zusammenhalt gefährdende Handlungen verpflichtet (Art 266). Als Verfassungsorgan wird ein nationaler Sicherheitsrat unter Vorsitz des Staatspräsidenten eingerichtet (Art 268). Dieser ist insbesondere vor Kriegserklärungen anzuhören (Art 269). Ähnliche Neutralitätspflichten normiert die neue Verfassung auch für die Polizei (Art 254 ff.).

\section{Justizwesen}

Die Dominanz der Regierungspartei der FRELIMO besteht auch im Bereich der staatlichen Judikative fort. Diese ist inzwischen zwar formell unabhängig, doch stellt die Ernennung der Obersten Richter durch den Präsidenten deren politische Neutralität in Frage. Außerdem bleibt das Justizsystem trotz diverser Reformansätze durch Ineffizienz und Korruption 
geprägt $^{33}$. Vor allem aber ist es von einer stukturellen Dysfunktionalität gekennzeichnet, bedingt durch mangelnde territoriale Durchdringung (lediglich 85 von 128 Distrikten verfügen über ein eigenes Gericht) sowie mangelnde personelle und materielle Ressourcen. Insgesamt sind in dem Land von knapp 20 Mio. Einwohnern nur 184 Richter tätig, von denen wiederum weniger als die Hälfte über einen Hochschulabschluss verfügt ${ }^{34}$. Nach Feststellung des Präsidenten des Obersten Gerichtshofs fehlt es bei vielen Richtern selbst an der Kenntnis grundlegender Rechtsbestimmungen oder elementarer Verfahrensgrundsätze $^{35}$. Der Überhang an Verfahren beträgt das Vierfache der Jahreskapazität und wächst trotz verbesserter Erledigungszahlen kontinuierlich weiter $\mathrm{an}^{36}$. Unabhängige Quellen schätzen, dass nur rund $10 \%$ der Bevölkerung effektiven Zugang zum staatlichen Justizwesen haben. Andererseits hat jüngst ein spektakulärer, von den Medien übertragener Prozess wegen Ermordung eines prominenten Journalisten (Carlos Cardoso) das öffentliche Ansehen der Justiz erheblich gestärkt, nachdem das Verfahren im Februar 2003 mit der Verurteilung der Angeklagten endete ${ }^{37}$.

Die neue Verfassung betont verstärkt die Unabhängigkeit der Justiz und regelt in diesem Zusammenhang Einzelheiten des Richteramts (Art 217 ff). In diesem Zusammenhang werden u.a. richterliche Nebenbeschäftigungen verfassungsrechtlich beschränkt (Art 219). Die rasche praktische Durchführbarkeit dieser Bestimmung muss allerdings bezweifelt werden, da örtliche Richter teilweise überhaupt keine finanzielle Unterstützung vom Staat erhalten $^{38}$. Der Auftrag der Richterschaft, die Bevölkerung zur Beachtung der Gesetze und zu einem gerechten und harmonischen Zusammenleben $\mathrm{zu}$ erziehen, wird auch durch die neue Verfassung fortgeschrieben (Art 213). Justizorganisatorisch bedeutsam und vor allem eine wichtige Stärkung der ordentlichen Gerichtsbarkeit ist, dass die verbindliche Einrichtung von Militär-, Zoll-, Steuer-, See- und Arbeitsgerichten künftig entfällt. Zwingend einzurichten sind künftig nur noch die ordentliche und die Verwaltungsgerichtsbarkeit (Art 223 (1)). Die übrigen Gerichtszweige sind fakultativ, dies gilt auch für die Einrichtung von

Cardoso Murder: Assassin's Refugee Hearing Postponed, Agencia de Informação de Moçambique vom 2.12.2004; Renamo Deputy Detained, Agencia de Informação de Moçambique vom 2.12.2004.

Huge Backlog of Cases in Mozambican Courts, Agencia de Informação de Moçambique vom 1.3.2005.

Huge Backlog of Cases in Mozambican Courts, Agencia de Informação de Moçambique vom 1.3.2005.

Huge Backlog of Cases in Mozambican Courts, Agencia de Informação de Moçambique vom 1.3.2005.

37 Cardoso Murder: Assassin's Refugee Hearing Postponed, Agencia de Informação de Moçambique vom 2.12.2004.

38

Bonate, Women's Land Rights in Mozambique, in: An-Na'im/Chandler, Women and Land in Africa, Atlanta 2002, Ziff. VI. 
Schieds- und Gemeinschaftsgerichten (Art 223 (2)). Die Zivil- und Strafgerichtsbarkeit bleibt in jedem Fall der ordentlichen Gerichtsbarkeit vorbehalten (Art 223 (4)). Militärgerichte können nur im Falle der Kriegserklärung und ausschließlich für Wehrdelikte eingerichtet werden (Art 224). Neu anerkannt wird ferner die Rolle der örtlichen Würdenträger bei der Streitschlichtung (Art 118 (2)), wie sie kraft einfachen Gesetzes etwa im Bereich des Bodenrechts bereits praktiziert wird ${ }^{39}$.

Außerdem wird nunmehr durch die Verfassung die Zusammensetzung und Funktionsweise des Obersten Richterrats geregelt. Er besteht aus dem Präsidenten und Vizepräsidenten des Obersten Gerichtshofs, zwei vom Staatspräsidenten ernannten Mitgliedern, fünf proportional vom Parlament gewählten Mitgliedern und sieben von ihren Kollegen gewählten Richtern der unterschiedlichen Kategorien (Art 221). Er ist für richterliche Personalentscheidungen zuständig und kann sich zur Justizpolitik äußern (Art 222).

\section{Verfassungsgerichtsbarkeit}

Einer der letzten Streitpunkte zwischen FRELIMO und RENAMO, welcher eine Verabschiedung bis fast zum letztmöglichen Zeitpunkt vor den Wahlen im Dezember 2004 verzögerte, betraf die Verfassungsgerichtsbarkeit ${ }^{40}$. Diese wurde erst 2003 eingerichtet und ist nicht nur für die Beurteilung der Verfassungsmäßigkeit von Gesetzen zuständig, sondern fungiert auch als Wahlgericht. Die RENAMO hatte versucht, eine Umbenennung des bisherigen Verfassungsrates (Art $180 \mathrm{ff}$ ) in einen Verfassungsgerichtshof durchzusetzen. Dabei ging es nicht nur um die Sicherung eines nominell gewichtigeren Status für das Gremium, sondern vor allem um die Schaffung eines Ansatzpunktes, um Falle eines Wahlsieges die FRELIMO-Dominanz auch in diesem Gremium aufbrechen zu können.

Bisher setzte sich das Gremium aus sieben Mitgliedern zusammen. Der Vorsitzende wurde vom Staatspräsident ernannt. Fünf weitere Mitglieder wurden nach der Mandatsverteilung im Parlament gewählt, was nach der bisherigen Sitzverteilung drei Vorschlagsrechte für die FRELIMO und zwei für RENAMO bedeutete. Das siebente Mitglied wurde von den übrigen sechs kooptiert, so dass sich eine Gewichtung von fünf der FRELIMO gegenüber zwei der RENAMO zuneigenden Mitgliedern ergibt. Der Konflikt wurde schließlich so aufgelöst, dass die Institution ihren bisherigen Namen fortführt und über die Tätigkeit als Wahlgericht bei den Wahlen im Dezember 2004 hinaus im Amt bleibt, die dort wirkenden Ent-

Art 21b des Gesetzes Nr. 17/97b über Bodennutzung mit Ministerrats-Dekret vom August 2000, vgl. Bonate, Women's Land Rights in Mozambique, in: An-Na'im/Chandler, Women and Land in Africa, Atlanta 2002, Ziff VI.

40

Constitutional Council Dispute Solved, Agencia de Informação de Moçambique vom 16.11.2004. 
scheidungsträger aber künftig als Richter bezeichnet werden (Art 241 ff.). Die Besetzung wird nunmehr durch die Verfassung selber geregelt, wobei materiell die einzige Änderung darin besteht, dass das siebente Mitglied künftig vom Obersten Richterrat gewählt wird (Art $242(1))$.

Außerdem werden die Kompetenzen des Gremiums ausgeweitet (Art 244). Hauptsächlich ist es wie bisher zuständig für Normenkontrollverfahren, Organstreitigkeiten und die Prüfung von Volksabstimmungen (Art 244 (1)). Außerdem wird es weiterhin als Wahlgericht tätig (Art 244 (2)). Neu hinzugekommen ist die Zuständigkeit für die Durchsetzung des Parteiengesetzes (Art 244 (2)). So kann es prüfen, ob die erforderliche Zahl von mindestens 2.000 ordnungsgemäßen Unterschriften erreicht wurde und ob die Namensgebung oder die verwendeten Symbole zur Gefahr der Verwechslung mit bereits etablierten Parteien führen $^{41}$. Ferner wird es in seine Kompetenz gelegt, den Staatspräsidenten für dauerhaft amtsunfähig zu erklären. Verfassungsbeschwerden einzelner Bürger sind hingegen auch nach der neuen Verfassung nicht statthaft. Gegen Entscheidungen des Verfassungsrates findet kein Rechtsmittel statt. Sie sind für alle Bürger und Einrichtungen verbindlich und werden im Amtsblatt veröffentlicht (Art 248).

\section{Wirtschaft}

a) Die erste Phase der Transformation

Der anhaltende Bürgerkrieg und Dürrekatastrophen hatten für die wirtschaftliche Entwicklung gravierende Folgen. Akuter Devisenmangel, stark rückläufige industrielle Produktion und die Krise des Agrarsektors führten zu Defiziten der Leistungsbilanz zwischen 20 und $24 \%$ des BSP $^{42}$. Einen Wendepunkt bedeutete das Scheitern des Versuchs der Anlehnung an den sozialistischen Wirtschaftsraum mit der Ablehnung des formalen Aufnahmeantrages Mosambiks zur Vollmitgliedschaft in den Rat für Gegenseitige Wirtschaftshilfe (RGW) im Jahre 1981. Mosambik erklärte daraufhin seinen Beitrittswunsch zum Lomé-Abkommen und unterzeichnete den sog Nkomati-Vertrag, durch welchen sich Mosambik und Südafrika zur Einstellung der Unterstützung für der Befreiungsbewegung im jeweils anderen Land (also RENAMO bzw ANC) verpflichteten. Dieser Kurswechsel wurde 1985/86 durch Beitrittsverhandlungen zur Weltbank und zum IWF sowie die Vorbereitung eines radikalen Strukturanpassungsprogramms in wirtschaftspolitischer Hinsicht 
nachvollzogen ${ }^{43}$. In Abkehr von der bisherigen zentral gelenkten, sozialistischen Planwirtschaft wurden die Preise aller Waren einschließlich der Grundnahrungsmittel frei gegeben, die Förderung der Staatsfarmen und Agrarkooperativen wurde zugunsten der privaten und familiären Landwirtschaft aufgegeben; die Unternehmen wurden auf kostendeckende, rentabilitätsorientierte Preisgestaltung umgestellt und erhielten weitgehende Autonomie in ihrer Betriebsführung. Das staatliche Außenhandelsmonopol wurde weitgehend gelockert und die Währung Metical wurde in kurzer Zeit um mehr als $1000 \%$ abgewertet.

\section{b) Die zweite Phase der Transformation}

Im Zuge des allgemeinen Transformationsprozesses wurde die bisherige Zentralverwaltungswirtschaft zielgerichtet liberalisiert. Dazu wurden u.a. sowohl einheimische wie ausländische Investitionen gesetzlich geschützt ${ }^{44}$ und die Mehrwertsteuer eingeführt. Außerdem gab es Steueranreize für Investitionen in den ärmsten Provinzen ${ }^{45}$.

Im Zuge dieser neuen Wirtschaftspolitik wurden die bisherigen Staatsunternehmen in großem Umfang privatisiert ${ }^{46}$. Privatwirtschaftliche Unternehmen stellen mittlerweile das Rückgrat der Volkswirtschaft dar. Die Tradition staatlicher Produktionsunternehmen aus der Zeit des Sozialismus setzt sich allerdings heute in der Form von staatlichen Kapitalbeteiligungen an neuen oder ehemals staatlichen, nun privatisierten Unternehmen fort. In zehn Unternehmen beträgt der staatliche Anteil zur Zeit noch mehr als $80 \%$. In circa 70 Unternehmen ist der Staat mit weniger als 50\% Anteilseigner (u. a. Banken BCM, Banco Austral). Ziel der Regierung ist es, diese Anteile weiter zu reduzieren. Da Direktoren und Besitzer privatisierter staatlicher Unternehmen häufig mit den Leitern der früheren Staatsbetriebe identisch sind oder aus der politischen Elite stammen (der neue Staatspräsident Guebuza gilt als einer der erfolgreichsten Geschäftsleute, u. a. mit Beteiligungen am Wasserversorgungsunternehmen Aguas de Moçambique) ${ }^{47}$, spricht man auch von "stillen Privatisierungen“. Die Konzentration des nationalen Produktions- und Anlagevermögens in den Händen weniger FRELIMO-Funktionäre und deren Familien unterstreicht die neo-patrimonialen Strukturen der neuen Wirtschaftsordnung. Hinzu kommt, dass sich die Privatisierung vor allem auf den Süden des Landes konzentrierte und die Politik der freien Marktwirtschaft vielerorts unzureichend umgesetzt wird. Dies hat zur Folge, dass in einigen Provin-

Programa da Restruturação Economica (PRE); vgl Proceminho, República de Moçambique Ficha de Mercado, Maputo 2002, 7 ff.

44

45

46

47 Gesetz Nr. 3/93 vom 24.6.1993.

Einzelheiten s. Proceminho, República de Moçambique - Ficha de Mercado, Maputo 2002, 14 ff.

Bertelsmann Transformation Index 2003, Länderbericht 2003, Gütersloh 2004, 4.

Msomi, SA Business Confident of Stability in Mozambique, Sunday Times (Johannesburg) vom 30.11.2004. 
zen immer noch Preiskontrollen stattfinden und keine steuerlichen Anreize für den Privatsektor gesetzt werden.

Zugleich wurde der Außenhandel schrittweise liberalisiert. In der Cashew-Industrie führte dies allerdings zum völligen Zusammenbruch des Sektors. Nach dem Ende des Bürgerkrieges wieder hergerichtetete Verarbeitungsanlagen mussten bereits nach kurzer Zeit wieder den Betrieb aufgeben, da der größte Teil der Ernte nach Indien zur dort weitaus billigeren Weiterverarbeitung exportiert wurde. Angesichts dieser Erfahrungen wurde die sich langsam von den Verwüstungen des Bürgerkrieges und die nachfolgenden Zerstörungen durch die Flutkatastrophe erholende Zuckerindustrie mit Schutzzöllen belegt ${ }^{48}$.

\section{c) Die gegenwärtige Lage}

Die allgemeine Stabilisierung hat auch zu einer insgesamt erfreulichen Wirtschaftsentwicklung geführt. Seit 1994 weist das BIP durchschnittliche Wachstumsraten von 8,4\% auf $^{49}$. Selbst nach einer wirtschaftlichen Stagnation im Jahr 2000 - bedingt durch die Überschwemmungskatastrophe im Zentrum und Süden des Landes - konnte Mosambik 2001 erneut ein Wirtschaftswachstum von 13,9\% aufweisen. Mit einem Volumen von 703,7 Mio. US-Dollar verdoppelte sich 2001 die Exportrate im Vergleich zum Vorjahr (364 Mio.). Verantwortlich hierfür war vor allem der Export von Aluminium durch die Aluminiumschmelze MOZAL im Wert von 383,5 Mio. (54,5\%). Steigende Exportraten schlugen sich darüber hinaus positiv in der Handelsbilanz nieder und reduzierten das Defizit von 789,3 Mio. in 2000 auf 447,1 Mio. in 2001.

Auch sind die Grundlagen für einen marktwirtschaftlichen Wettbewerb inzwischen vorhanden, jedoch bestehen strukturelle Hindernisse insbesondere in den Provinzen im Zentrum und Norden des Landes zur Etablierung eines echten Wettbewerbs privater Unternehmen (mangelnde Infrastruktur, kaum einheimische oder ausländische Investitionen) ${ }^{50}$. Das Augenmerk der Regierung lag bisher vor allem auf wirtschaftlichen Großprojekten, wie der Aluminiumschmelze MOZAL. Monopole existieren vor allem noch in den Bereichen nationaler Flugverkehr, Telekommunikation und Energieversorgung. Eine Herausbildung eines soliden Mittelstandes fand bislang nicht statt. Die rechtlichen Rahmenbedingungen für die Legalisierung von Klein- und Kleinstunternehmen sind noch immer unzureichend,

48

49

50

Bertelsmann Transformation Index 2003, Länderbericht 2003, Gütersloh 2004, 13, 20.

Bertelsmann Transformation Index 2003, Länderbericht 2003, Gütersloh 2004, 12, 15; International Monetary Fund, Republic of Mozambique: Poverty Reduction Strategy Paper Progress Report, IMF Country Report 04/135, Washington 2004, 33 ff.; Proceminho, República de Moçambique - Ficha de Mercado, Maputo 2002, 10 ff., 17 ff.

Bertelsmann Transformation Index 2003, Länderbericht 2003, Gütersloh 2004, 12. 
wie auch die Investitionsbedingungen in den meisten Provinzen. Auf der Basis eines neuen Mikrofinanzgesetzes beginnen sich mittlerweile allerdings zahlreiche der von internationalen Nichtregierungsorganisationen finanzierten Mikrofinanzprogramme in permanente mosambikanische Mikrofinanzinstitutionen zu transformieren. Für mittlere, kleine und Mikrounternehmen stellen sie die einzige Kreditmöglichkeit dar. Einen wirtschaftlichen Hemmschuh bildet auch die Schwäche des Justizsystems im Handels- und Arbeitsrecht. Rund $80 \%$ der Bevölkerung sind nach wie vor in der Landwirtschaft tätig, welche vielfach als Subsistenzwirtschaft betrieben wird ${ }^{51}$.

Das Banken- und Finanzsystem ist überwiegend in privatwirtschaftlicher Hand, obgleich die Regierung bei einigen Banken Anteilseigner ist ${ }^{52}$. Ausländische Banken haben ungehinderten Zugang zum Markt. Die jüngste Krise im Bankensektor (Verlust von circa 400 Mio. US-Dollar durch Betrügereien in der Privatisierung der Banco Comercial de Moçambique (BCM) und weitere Verluste durch ungedeckte Kredite bei der Banco Austral) offenbaren nicht nur die unzureichende Bankenaufsicht ${ }^{53}$, sondern führten letztlich mit der Fusion von BCM und Banco Internacional de Moçambique auch zur weiteren Konzentration im Bankengewerbe. Die mosambikanische Zentralbank Banco de Moçambique ist rechtlich unabhängig und besitzt sowohl Verwaltungs- als auch Finanzautonomie. Der Direktor und sein Stellvertreter werden jedoch vom Staatspräsidenten und der Vorstand vom Premierminister ernannt. Die Währung Metical ist frei konvertierbar und unterliegt nicht der Kontrolle durch die Zentralbank.

Problematisch ist auch die Anwendung der Regelungen für den Erwerb und Besitz privaten Eigentums ${ }^{54}$. Alle Bodenflächen gehören dem Staat und können lediglich für einen Zeitraum von maximal 50 Jahren gepachtet werden. Das in einem partizipativen Prozess entstandene Bodenrecht, welches grundsätzlich die Rechte der bisherigen Landnutzer sichert, sollte eigentlich Konflikten von vornherein vorbeugen ${ }^{55}$. In der Praxis erfolgt die Landzumessung jedoch weitgehend ohne institutionalisierte Kontrolle. Immer wieder kommt es zu illegalen Enteignungen von Grundstücken in touristisch oder agrarwirtschaft-

International Monetary Fund, Republic of Mozambique: Poverty Reduction Strategy Paper Progress Report, IMF Country Report 04/135, Washington 2004, 25, 80 ff.; Proceminho, República de Moçambique - Ficha de Mercado, Maputo 2002, 8 ff.

Bertelsmann Transformation Index 2003, Länderbericht 2003, Gütersloh 2004, 13.

International Monetary Fund, Republic of Mozambique: Poverty Reduction Strategy Paper Progress Report, IMF Country Report 04/135, Washington 2004, 46. 
lich lukrativen Gebieten. Nutznießer sind häufig Vertreter des Staates bzw. der politischen Elite $^{56}$.

Als besonders armes Land ist Mosambik in großem Umfang Empfänger ausländischer Entwicklungshilfe. Nach wie vor wird fast die Hälfte der Staatsausgaben aus dem Ausland finanziert ${ }^{57}$. Ein eigenständiger finanzieller Gestaltungsspielraum besteht kaum.

Die wirtschaftspolitischen Aussagen der neuen Verfassung (Art 96 ff.) lassen den zwischenzeitlichen Reformprozess kaum erkennen. Auch in den neu eingefügten Bestimmungen steht vor allem die staatliche Lenkungsfunktion im Vordergrund. Nach einer neu aufgenommenen Grundsatzbestimmung hat die staatliche Wirtschaftspolitik die Grundlagen für die Entwicklung des Landes zu schaffen, die Lebensbedingungen der Bevölkerung zu verbessern und zur staatlichen Einheit beizutragen (Art 96). Der Staat garantiert außerdem die Verteilung des nationalen Reichtums und würdigt die Rolle der produktiven Regionen. Weiten Auslegungsspielraum eröffnet die Aufzählung wirtschaftspolitischer Grundsätze, welche das Wirken der Marktkräfte und die Entfaltung unternehmerischer Initiative zwar erwähnen, dies aber unverbunden neben weitere Grundsätze wie den Schutz des kooperativen und sozialen Sektors, die Koexistenz staatlicher und privater Unternehmungen sowie die Rolle des Staates als Förderer der wirtschaftlichen und sozialen Entwicklung stellt (Art 97). Die staatliche Wirtschaftspolitik hat auch zur Lösung der grundlegenden Probleme des Volkes und zur Verringerung sozialer und regionaler Ungleichgewichte beizutragen (Art 101).

Eigentum und Erbrecht bleiben im bisherigen Rahmen gewährleistet, eine Ausweitung des grundgesetzlichen Schutzes vor Enteignungen ist nicht erfolgt (Art 82, 83). Zur Grundlage der wirtschaftlichen Entwicklung wird weiterhin die Landwirtschaft erklärt (Art 103), während die Industrie Impulsgeber sein soll (Art 104). Ferner wird wie bisher die grundlegende Rolle der Familienbetriebe anerkannt und - trotz der inzwischen erfolgten Neuausrichtung der staatlichen Landwirtschaftpolitik - ihre Umwandlung in "fortschrittliche Organisationsformen" gefördert (Art 105). Besonders gefördert werden sollen auch weiterhin die Kleinbetriebe (Art 106). Dem privaten Unternehmertum bleibt die Möglichkeit einer "aktiven Teilhabe" an der wirtschaftlichen Entwicklung des Landes eröffnet (Art 107 (1)). Außerdem haben sie jetzt Anspruch auf eine staatliche Wirtschaftsförderung, besonders in ländlichen Gebieten (Art 107 (2)). Ausländische Investitionen bleiben im Rahmen

Maputo City Council Acts On Illegal Land Sales, Agencia de Informação de Moçambique vom 25.1.2001. 
der staatlichen Wirtschaftspolitik möglich und sind auf allen wirtschaftlichen Gebieten zulässig, soweit dort kein staatliches Monopol besteht (Art 108). Hier bleibt es bedeutsam, dass die Rohstoffe und natürlichen Ressourcen exklusives Staatsvermögen bilden (Art 98), allerdings ist der Staat verpflichtet, deren Nutzung zu fördern (Art 102). Ferner bleiben sämtliche Bodenflächen in staatlichem Eigentum (Art 109). Wie bisher sind Bodenrechte aufgrund Erbschaft oder tatsächlicher Nutzung zu schützen, soweit nicht das Gesetz etwas anderes bestimmt oder die Fläche rechtmäßig einer anderen Person übertragen wurde (Art 111). Der Staat ist ferner wie bisher beauftragt, für eine gerechte Verteilung der Früchte der Werktätigkeit zu kämpfen (Art 112 (2)). Außerdem hat er sich künftig für das Prinzip "Gleicher Lohn für gleiche Arbeit" einzusetzen.

Die wirtschaftspolitische Modernisierung konzentriert sich so auf das völlig neu geschaffene Kapitel über staatliche Finanzpolitik (Art 126 ff.). Danach ist das Finanzwesen so zu organisieren, dass die private Vermögensbildung geschützt wird (Art 126). Steuern sind wie bisher nach dem Kriterium der sozialen Gerechtigkeit zu erheben (Art 100). Als weitere Grundsätze des öffentlichen Finanzwesens sind außerdem die Sicherung ausreichender staatlicher Einkünfte, die Verwirklichung der Ziele der Wirtschaftspolitik und die gerechte Verteilung des Wohlstands hinzugekommen (Art 127 (1)). Die rückwirkende Belastung mit Steuern wird verboten (Art 127 (5)). Als zentrales Instrument der staatlichen Wirtschaftspolitik erhalten die 5-Jahrespläne Verfassungsrang (Art 128 ff.). Sie haben das nachhaltige Wachstum zu fördern und Ungleichgewichte zwischen Regionen bzw. zwischen Stadt und Land abzubauen (Art 128 (1)). Sie sind vom Parlament zu verabschieden und durch die staatliche Haushaltsführung umzusetzen (Art 129 (2), (3)). Als weitere Elemente treten die Normierung von Grundsätzen für die staatliche Haushaltführung (Art 130), Rechnungsprüfung (Art 131) und die Zentralbank (Art 132) hinzu.

\section{Bildung, Gesundheit, Sozialwesen, Umwelt}

Der Stabilisierungsprozess der letzten zehn Jahre hat nicht nur makroökonomisch, sondern auch für die Lebenssituation der Menschen erfreuliche Ergebnisse gezeigt ${ }^{58}$. Dies spiegelt der UNDP-HDI-Index, welcher zwischen 1994 und 2001 von 0,310 kräftig auf 0,378 gestiegen ist. Dennoch zählt Mosambik noch immer zu den am geringsten entwickelten Ländern der Erde; im UNDP-Ranking liegt es an viertletzter Stelle. Hinzu kommen enorme regionale Ungleichgewichtigkeiten besonders zwischen der relativ gut entwickelten Hauptstadt Maputo im Süden und strukturschwachen Gebieten wie etwa die Zambezia-Provinz national Monetary Fund, Republic of Mozambique: Poverty Reduction Strategy Paper Progress Report, IMF Country Report 04/135, Washington 2004, 22 ff. 
im Norden. Mit Ausnahme der Nordprovinzen (Zambezia, Nampula, Cabo Delgado) wiesen die ländlichen Regionen zuletzt allerdings ein überproportionales Wachstum auf. Soziale Exklusionen sind quantitativ und qualitativ stark ausgeprägt und strukturell verfestigt. 69,4\% der Bevölkerung leben unter der Armutsgrenze von 0,40 US-Dollar pro Tag. Die Analphabetenrate liegt bei 56,7 \%; sie ist damit eine der höchsten Afrikas ${ }^{59}$.

Im Kontext einer nachhaltigen Armutsbekämpfung versucht die mosambikanische Regierung das Bildungssystem zielgerichtet zu verbessern ${ }^{60}$. Dabei konzentriert man sich auf den Zugang, die Qualität und auch die Kapazität des Bildungssektors. Zur Zeit stehen 23,1\% des Etats für Bildung zur Verfügung. Aufgrund der allgemeinen Finanzschwäche ist die Umsetzung des ambitionierten strategischen Entwicklungsplans für den Bildungsbereich dennoch in Frage gestellt. Insbesondere die Qualität der Bildung wird als Entwicklungshindernis wahr genommen. Sie verursacht bereits beim Übergang von der Grundschule in die Sekundarstufe als auch später beim Übergang zur höheren Bildung gravierende Probleme. Diskutiert werden sowohl die Diversifizierung der Angebote als auch die Einbeziehung privater Träger. Private Investitionen sind allerdings bislang lediglich im Hochschulbereich mit der Katholischen Universität und den Hochschulen Instituto Superior Politecnico e Universario (ISPU) sowie Instituto Superior de Ciências e Tecnologia de Moçambique (ISCTM) von Bedeutung.

Für die Mehrzahl der Bevölkerung im erwerbsfähigen Alter stellt aufgrund der Beschäftigung im informellen Sektor oder in der Subsistenzwirtschaft die Familie die einzige soziale Absicherung dar ${ }^{61}$. Tragfähige soziale familiäre Netze zur Kompensation von Armut sind bei der Zahl der unter der Armutsgrenze Lebenden jedoch wenig leistungsfähig. Hinzu kommt, dass bestehende Familienstrukturen angesichts der HIV-/Aids-Epidemie zunehmend auseinander brechen ${ }^{62}$. Schätzungen gehen davon aus, dass sich die aktuelle Zahl von 500.000 Aids-Waisen im Jahre 2010 auf 1,5 Mio. erhöht haben wird. Das 1988 gegründete Sozialversicherungsinstitut INSS bietet zur Zeit circa 321.000 registrierten Arbeitern eine formale Absicherung ${ }^{63}$.

Bertelsmann Transformation Index 2003, Länderbericht 2003, Gütersloh 2004, 12, 16; International Monetary Fund, Republic of Mozambique: Poverty Reduction Strategy Paper Progress Report, IMF Country Report 04/135, Washington 2004, 23, $51 \mathrm{ff}$.

International Monetary Fund, Republic of Mozambique: Poverty Reduction Strategy Paper Progress Report, IMF Country Report 04/135, Washington 2004, 60 ff.

International Monetary Fund, Republic of Mozambique: Poverty Reduction Strategy Paper Progress Report, IMF Country Report 04/135, Washington 2004, 25 ff., 64 ff.

Proceminho, República de Moçambique - Ficha de Mercado, Maputo 2002, 13. 
Die Aussichten für eine positive Entwicklung sind im Bildungsbereich, wie auch für die allgemeine Lebenserwartung, überschattet von den Auswirkungen der AIDS-Epidemie ${ }^{64}$. Die Lebenserwartung für Mosambikaner stieg zwar von 41,7 Jahren im Jahre 1994 auf 44,6 im Jahre 2000. Aber eine Infektionsrate von 16\% (2001) der erwachsenen Bevölkerung hat bereits zu einem Absinken der Lebenserwartung auf rund 38 Jahre mit weiter kräftig fallender Tendenz geführt.

Unter dem langanhaltenden Bürgerkrieg litt in den Nationalparks vor allem die Fauna, während die Küstengebiete vor touristischer Erschließung bewahrt blieben. Mit dem Friedensschluss hat sich beides inzwischen umgekehrt. Ein erhebliches Problem stellten in den letzten Jahren wiederholt Überschwemmungen dar ${ }^{65}$.

Die neue Verfassung schreibt die bisherigen Regelungen für eine staatliche Familien- (Art 119, 120), Frauen- (Art 122) und Jugendpolitik (Art 123) bei weiteren Konkretisierungen im Detail fort. Neu hinzugetreten sind entsprechende Bestimmungen für Kinder (Art 121), Senioren (Art 124) und Behinderte (Art 125).

Deutlich erweitert wurden die Aussagen zum Bildungsbereich. Der Staat ist weiterhin für die Einrichtung eines staatlichen Bildungswesens und eine nationale Bildungsstrategie zuständig, welche der nationalen Einheit, der Ausrottung des Analphabetismus, der Herrschaft von Wissenschaft und Technik sowie der ethischen und bürgerschaftlichen Erziehung zu dienen hat (Art 113 (1), (2)). Zusätzlich wird jetzt die politische und weltanschauliche Neutralität des öffentlichen Bildungswesens postuliert (Art 113 (3), (5)), und vor allem werden private Bildungsträger ausdrücklich zugelassen (Art 113 (4)). Neu ist ferner die Regelung des Hochschulwesens (Art 114). Hochschulen in öffentlicher Trägerschaft haben eigenständige Rechtspersönlichkeit und genießen vorbehaltlich der staatlichen Qualitätssicherung wissenschaftliche, pädagogische, finanzielle und administrative Autonomie (Art 114 (2)). Eine wesentliche Einschränkung dieser Autonomie liegt allerdings in der Befugnis des Staatspräsidenten zur Ernennung der Hochschulleitung (Art 160 (2)). Private Hochschulen haben Anspruch auf staatliche Finanzierung (Art 114 (3)).

Auch im Gesundheitsbereich führt die neue Verfassung im wesentlichen die bisherigen Bestimmungen über die Einrichtung eines für alle Bürger zuständigen staatlichen Gesundheitswesens fort (Art 116). Diese Linie wird insofern noch verstärkt, als der gleiche Zugang zu Gesundheitsdienstleistungen garantiert wird (Art 116 (4)). Der Staat lenkt ferner den Arzneimittelmarkt (Art 116 (5)) und regelt die Tätigkeit privater Gesundheitsanbieter (Art 
116 (6)). Erheblich detaillierter als bisher wird auch die staatliche Umweltpolitik abgehandelt (Art 117). So ist in allen Politikbereichen ein Umwelt-Mainstreaming durchzuführen, und es wird die Bedeutung der Umwelterziehung hervorgehoben (Art 117 (2)). Außerdem trifft den einzelnen Bürger eine Pflicht zum Umweltschutz (Art 45).

\section{Verfassungsänderungen}

Eine Stärkung der Rechtsstaatlichkeit bedeutet die Umgestaltung der bisher rein prozeduralen Regelungen zur Verfassungsänderung (Art 198 ff). Neu ist vor allem, dass wesentliche materielle Grundsätze der Verfassung dauerhaft festgeschrieben wurden. Hierzu gehören die Trennung von Staat und Kirche, die republikanische Staatsform, die Achtung der Bürger- und Grundrechte, die Grundsätze der allgemeinen und geheimen Wahl, politische Pluralität und das Recht auf eine demokratische Opposition, Gewaltenteilung und Unabhängigkeit der Justiz, das Recht auf Gründung von Gewerkschaften, die lokale Selbstverwaltung sowie der Schutz der Staatsangehörigkeit (Art 292 (1)). Verfassungsänderungen in diesen Bereichen müssen außerdem obligatorisch dem Volk zur Abstimmung vorgelegt werden (Art 292 (2)). Jede Verfassungsänderung erfordert außerdem eine Mehrheit von zwei Dritteln der Mitglieder des Parlamentes (Art 295). Liegt die letzte Verfassungsänderung weniger als fünf Jahre zurück, ist eine Verfassungsänderung nur ausnahmsweise und nur mit einer Mehrheit von drei Vierteln möglich (Art 293). Nach jeder Verfassungsänderung ist zusammen mit dem Änderungsgesetz die konsolidierte Fassung der geänderten Verfassung zu veröffentlichen (Art 296).

\section{Ausblick}

Die neue Verfassung zeigt ein getreues Abbild des gegenwärtigen Standes im Transformationsprozess. Insbesondere schlägt sich ein verändertes Verständnis für die Bedeutung von Rechtsstaatlichkeit für eine geordnete gesellschaftliche Entwicklung im Verfassungstext nieder. Dies zeigt sich nicht nur in der Ausweitung des Grundrechtsschutzes, sondern beispielsweise auch in der weiteren Ausgestaltung des Justizwesens, in der Schaffung des Amtes eines Ombudsmans, im Staatsangehörigkeitsrecht und in der Neuordnung der Regelungen über Volksabstimmungen. Eine gewisse gesellschaftliche Öffnung spiegelt sich auch in dem erweiterten Spielraum für kirchliche Aktivitäten, der Anerkennung traditioneller Autoritäten oder auch in der Festschreibung der Hochschulautonomie. Andererseits 
wurde die Chance verpasst, sich entsprechend der staatlichen Wirtschaftspolitik ${ }^{66}$ auch verfassungsrechtlich als Standort mit guten Rahmenbedingungen für Investitionen zu präsentieren. Vielmehr blieb es im Wesentlichen bei einer Fortschreibung des bisherigen, den Übergang von einer Zentralverwaltungs- in eine Marktwirtschaft markierenden Zustand. Erschwerend kommt hinzu, dass in einer Reihe von Punkten der populistischen Versuchung nachgegeben wurde und bei der derzeitigen Wirtschaftslage nicht einlösbare soziale Grundrechte normiert wurden, welche wie etwa das neue Verbraucherschutzgrundrecht neben dem Staat auch die Wirtschaft unmittelbar binden sollen. Wirtschaftlich hemmend dürfte sich auch die großzügige Einführung von Popular- und Verbandsklagen zumal im Zusammenspiel mit einer schon heute überforderten Justiz - auswirken.

Kaum betroffen von der Verfassungsänderung ist die staatliche Machtverteilung. Auch hier lässt sich ein vor allem aus der Interessenlage der Hauptbeteiligten erklärliches Festhalten am bisherigen System staatlicher Machtkonzentration beobachten. Trotz leicht erweiterter Kompetenzen des Parlaments und Ausbaus der Institutionen der lokalen Selbstverwaltung bleibt das Präsidentenamt das alles dominierende Machtzentrum. Zudem stellt die von neopatrimonialen Netzwerken und Korruption durchzogene politische Kultur die dauerhafte Konsolidierung demokratischer Strukturen nach wie vor in Frage ${ }^{67}$. Andererseits ist es auch der parlamentarischen Opposition, welche sich seit den verlorenen Wahlen des Jahres 1999 primär durch destruktives Verhalten und Boykottaktionen profiliert, nicht gelungen, ein glaubwürdiges Angebot politischer Alternativen zu präsentieren. Diese Paralyse des dringend notwendigen politischen Dialoges setzte sich nach den Wahlen im Dezember 2004 nahtlos fort. Die gestärkte Stellung des Staatspräsidenten nach diesen Wahlen und dessen persönliche Verflechtung mit dem Wirtschaftsektor seines Landes lassen eine weitere Verfestigung patrimonialer Strukturen befürchten. Sie könnte aber auch Chancen bieten, aus dieser Position der Stärke heraus den Aufbau einer lebendigen Zivilgesellschaft, die Stärkung der Unabhängigkeit der Justiz und die Befreiung der Wirtschaft von staatlicher Fehllenkung und staatlich subventionierter Konkurrenz voranzubringen.

Der Sieger der Präsidentschaftswahlen 2004 bezeichnete dies unmittelbar nach der Wahl gleichwohl als das Hauptanliegen seiner Mandatsperiode, Election Campaign Ends, Agencia de Informação de Moçambique vom 30.11.2004. 